Article

\title{
On some Integral Representations of Certain G-Functions
}

\section{Seemon Thomas}

Received: 3 November 2015; Accepted: 23 December 2015; Published: 31 December 2015 Academic Editor: Hans J. Haubold

Department of Statistics, St Thomas College Pala, Kerala 686574, India; palaseemon@gmail.com; Tel.: +91-949-532-5341

Abstract: This is a brief exposition of some statistical techniques utilized to obtain several useful integral equations involving G-functions.

Keywords: generalized type-1 Dirichlet model; marginal density; Meijer's G-function; moments

\section{Introduction}

Thomas and George [1] introduced a generalized type-1 Dirichlet model having several mathematical and statistical properties. The density has been derived from the property of the following ratios:

Let $x_{1}, \ldots, x_{k}$ be such that $0<x_{i}<1, i=1, \ldots, k, 0<x_{1}+\cdots+x_{k}<1$ and let

$$
\frac{x_{1}}{x_{1}+x_{2}}, \frac{x_{1}+x_{2}}{x_{1}+x_{2}+x_{3}}, \cdots, \frac{x_{1}+\cdots+x_{k-1}}{x_{1}+\cdots+x_{k}}, x_{1}+\cdots+x_{k}
$$

be independently distributed as type- 1 beta with parameters $\left(\alpha_{1}, \alpha_{2}\right),\left(\alpha_{1}+\alpha_{2}+\beta_{2}, \alpha_{3}\right),\left(\alpha_{1}+\alpha_{2}+\right.$ $\left.\alpha_{3}+\beta_{2}+\beta_{3}, \alpha_{4}\right), \ldots,\left(\alpha_{1}+\cdots+\alpha_{k}+\beta_{2}+\cdots+\beta_{k}, \alpha_{k+1}\right)$ respectively. Then $\left(x_{1}, \ldots, x_{k}\right)$ has the density function of the following form:

$$
f\left(x_{1}, \ldots, x_{k}\right)=c_{k} x_{1}^{\alpha_{1}-1} \ldots x_{k}^{\alpha_{k}-1}\left(x_{1}+x_{2}\right)^{\beta_{2}} \ldots\left(x_{1}+\cdots+x_{k}\right)^{\beta_{k}}\left(1-x_{1}-\cdots-x_{k}\right)^{\alpha_{k+1}-1} .
$$

Obviously it is a generalization of type-1 Dirichlet probability model. The normalizing constant $c_{k}$ can be evaluated as

$$
\begin{aligned}
c_{k}= & \frac{\Gamma\left(\alpha_{1}+\alpha_{2}\right)}{\Gamma\left(\alpha_{1}\right) \Gamma\left(\alpha_{2}\right) \ldots \Gamma\left(\alpha_{k+1}\right)} \frac{\Gamma\left(\alpha_{1}+\alpha_{2}+\alpha_{3}+\beta_{2}\right)}{\Gamma\left(\alpha_{1}+\alpha_{2}+\beta_{2}\right)} \ldots \\
& \times \frac{\Gamma\left(\alpha_{1}+\cdots+\alpha_{k}+\beta_{2}+\cdots+\beta_{k-1}\right)}{\Gamma\left(\alpha_{1}+\cdots+\alpha_{k-1}+\beta_{2}+\cdots+\beta_{k-1}\right)} \frac{\Gamma\left(\alpha_{1}+\cdots+\alpha_{k+1}+\beta_{2}+\cdots+\beta_{k}\right)}{\Gamma\left(\alpha_{1}+\cdots+\alpha_{k}+\beta_{2}+\cdots+\beta_{k}\right)}
\end{aligned}
$$

for $\Re\left(\alpha_{j}\right)>0, j=1, \ldots, k+1, \Re\left(\alpha_{1}+\cdots+\alpha_{j}+\beta_{2}+\cdots+\beta_{j}\right)>0, j=2, \ldots, k$, where $\Re$ denotes the real part of $(\cdot)$. For more properties of the Model (1) one may refer Thomas and George [1]. Note that

$$
x_{1}=\frac{x_{1}}{x_{1}+x_{2}} \frac{x_{1}+x_{2}}{x_{1}+x_{2}+x_{3}} \ldots \frac{x_{1}+\cdots+x_{k-1}}{x_{1}+\cdots+x_{k}}\left(x_{1}+\cdots+x_{k}\right)
$$

is structurally a product of $k$ independent real variables and its density can be written in terms of a $G$-function of the type $G_{k, k}^{k, 0}(\cdot)$. The majority of the established special functions can be represented in terms of the G-function. A notable property of $G$-functions is the closure property. The closure property implies that whenever a function is expressible as a G-function of a constant multiple of 
some constant power of the function argument, the derivative and the antiderivative of this function are expressible so too. A general G-function is defined as the following Mellin-Barnes integral:

$$
\begin{aligned}
G_{p, q}^{m, n}(z) & =G_{p, q}^{m, n}\left(\left.z\right|_{b_{1}, \ldots, b_{q}} ^{a_{1} \ldots, a_{p}}\right) \\
& =\frac{1}{2 \pi i} \int_{\mathcal{L}} \frac{\left\{\prod_{j=1}^{m} \Gamma\left(b_{j}+s\right)\right\}\left\{\prod_{j=1}^{n} \Gamma\left(1-a_{j}-s\right)\right\}}{\left\{\prod_{j=m+1}^{q} \Gamma\left(1-b_{j}-s\right)\right\}\left\{\prod_{j=n+1}^{p} \Gamma\left(a_{j}+s\right)\right\}} z^{-s} \mathrm{~d} s
\end{aligned}
$$

where $i=\sqrt{(-1)}$ and $\mathcal{L}$ is a suitable contour.

The existence of different types of contours, properties and applications of $G$-functions are available in Mathai and Haubold [2].

\section{Integral Representations}

All the random variables considered above take values in $[0,1]$ and hence the density functions can be uniquely determined by their moments. For arbitary $t$, we have

$$
E\left(x_{1}^{t}\right)=c_{k}^{\prime} \prod_{j=1}^{k} \frac{\Gamma\left(\alpha_{1}+\cdots+\alpha_{j}+\beta_{2}+\cdots+\beta_{j}+t\right)}{\Gamma\left(\alpha_{1}+\cdots+\alpha_{j+1}+\beta_{2}+\cdots+\beta_{j}+t\right)}
$$

where

$$
c_{k}^{\prime}=\prod_{j=1}^{k} \frac{\Gamma\left(\alpha_{1}+\cdots+\alpha_{j+1}+\beta_{2}+\cdots+\beta_{j}\right)}{\Gamma\left(\alpha_{1}+\cdots+\alpha_{j}+\beta_{2}+\cdots+\beta_{j}\right)} .
$$

Note that the moments of the product of independent random variables are the products of the respective moments. Treating Equation (3) as a Mellin transform of the density of $x_{1}$, the density is available by the inverse Mellin transform. Thus, the density of $x_{1}$ is the following:

$$
\begin{aligned}
g\left(x_{1}\right) & =c_{k}^{\prime} x_{1}^{-1} \frac{1}{2 \pi i} \int_{\mathcal{L}} \frac{\Gamma\left(\alpha_{1}+t\right)}{\Gamma\left(\alpha_{1}+\alpha_{2}+t\right)} \cdots \frac{\Gamma\left(\alpha_{1}+\cdots+\alpha_{k}+\beta_{2}+\cdots+\beta_{k}+t\right)}{\Gamma\left(\alpha_{1}+\cdots+\alpha_{k+1}+\beta_{2}+\cdots+\beta_{k}+t\right)} x_{1}^{-t} \mathrm{~d} t \\
& =c_{k}^{\prime} x_{1}^{-1} G_{k, k}^{k, 0}\left[\left.x_{1}\right|_{\alpha_{1}, \cdots, \alpha_{1}+\cdots+\alpha_{k}+\beta_{2}+\cdots+\beta_{k}} ^{\alpha_{1}+\alpha_{2}, \ldots, \alpha_{1}+\cdots+\alpha_{k}+\beta_{2}}\right]
\end{aligned}
$$

for $0<x_{1}<1$ and zero elsewhere.

\section{Proposition 1.}

$$
\begin{gathered}
G_{k, k}^{k, 0}\left[\left.x_{1}\right|_{\alpha_{1}, \ldots, \alpha_{1}+\cdots+\alpha_{k}+\beta_{2}+\cdots+\beta_{k}} ^{\alpha_{1}+\alpha_{2}, \ldots, \alpha_{1}+\cdots+\alpha_{k+1}+\beta_{2}+\cdots+\beta_{k}}\right]= \\
\frac{x_{1}}{\Gamma\left(\alpha_{2}\right) \Gamma\left(\alpha_{3}\right) \ldots \Gamma\left(\alpha_{k+1}\right)} \int_{0}^{1-x_{1}} \int_{0}^{1-x_{1}-x_{2}} \cdots \int_{0}^{1-x_{1}-\cdots \cdot-x_{k-1}} x_{1}^{\alpha_{1}-1} \ldots x_{k}^{\alpha_{k-1}} \\
\times\left(x_{1}+x_{2}\right)^{\beta_{2}} \ldots\left(x_{1}+\cdots+x_{k}\right)^{\beta_{k}}\left(1-x_{1}-\cdots-x_{k}\right)^{\alpha_{k+1}^{-1}} \mathrm{~d} x_{k} \mathrm{~d} x_{k-1} \ldots \mathrm{d} x_{2} .
\end{gathered}
$$

Proof. The result follows by equating Equation (4) with the marginal density of $x_{1}$ obtained by integrating out $x_{2}, \ldots, x_{k}$ from the joint density of $x_{1}, \ldots, x_{k}$ given in Equation (1).

Let $X_{j}, j=1, \ldots, k$ be an ordered set of points in the Euclidean $n$-space $\mathcal{R}^{n}, n \geq k$. Let $O$ denotes the origin of a rectangular coordinate system. Now the $1 \times n$ vector $X_{j}$ can be considered as a point in $\mathcal{R}^{n}$. If $X_{1}, \ldots, X_{k}$ are linearly independent then the convex hull generated by these $k$-points 
almost surely determine a $k$-parallelotope in $\mathcal{R}^{n}$ with the sides $\overrightarrow{O X_{1}}, \ldots, \overrightarrow{O X_{k}}$. The random volume or $\mathrm{k}$-content $\nabla k, n$ of this random parallelotope is given by

$$
\nabla k, n=\left|X X^{\prime}\right|^{1 / 2}
$$

where

$$
X=\left(\begin{array}{c}
X_{1} \\
\vdots \\
X_{k}
\end{array}\right)
$$

is a matrix of order $k \times n, X^{\prime}$ is the transpose of $X$ and $|(\cdot)|$ denotes the determinant of $(\cdot)$. The classical approach to random points and random volumes consists in looking at independently distributed isotropic random points and dealing with random geometric configurations with the help of techniques from differential and integral geometry. Mathai [3] looked into random volumes under a more general structure by deleting the assumptions of independence and isotropy. Mathai [3] has shown that if the $k \times n, n \geq k$, real random matrix $X$ of full rank $k$ has the density:

$$
f(X)=C\left|X X^{\prime}\right|^{\alpha}\left|I-X X^{\prime}\right|^{\beta-\frac{k+1}{2}}
$$

for $0<X X^{\prime}<I$, then the probability distribution of $\nabla_{k, n}^{2}=\left|X X^{\prime}\right|$ has the following structure:

$$
\nabla_{k, n}^{2} \stackrel{\mathrm{d}}{=} \prod_{j=1}^{k} \text { type- } 1 \text { beta }\left[\alpha+\frac{1}{2}(n+1-j), \beta\right] .
$$

Thus, it is possible to express the density of $\nabla_{k, n}^{2}$ as a marginal density of $x_{1}$ obtained from the joint density given in Equation (1) with specific set of parameters. The notion $\nabla_{k, n}^{2}$ has application in the study of variance of multivariate distributions. More details on random volumes may be seen from Mathai [3]. Thomas and Mathai [4] expressed the density of $\nabla_{k, n}^{2}$ as a marginal density of $x_{1}$ in the Model (1) with parameters as

$$
\alpha_{1}=\alpha+\frac{n}{2}, \alpha_{2}=\alpha_{3}=\cdots=\alpha_{k+1}=\beta, \quad \beta_{2}=\cdots=\beta_{k}=-\left(\beta+\frac{1}{2}\right) .
$$

Now let us consider the Gaussian or ordinary hypergeometric function ${ }_{2} F_{1}(a, b ; c ; z)$ which is a special function represented by the hypergeometric series:

$$
{ }_{2} F_{1}(a, b ; c ; z)=\sum_{r=0}^{\infty} \frac{(a)_{r}(b)_{r}}{(c)_{r}} \frac{z^{r}}{r !}
$$

where

$$
(a)_{r}=(a+r-1)(a+r-2) \cdots(a)=\frac{\Gamma(a+r)}{\Gamma(a)} ;(a)_{0}=1, a \neq 0
$$

when $\Gamma(a)$ is defined.

\section{Proposition 2.}

$$
{ }_{2} F_{1}\left(\beta, \beta+\frac{1}{2} ; 2 \beta ; 1-x\right)=2^{2 \beta-1} x^{-\frac{1}{2}}\left(1+x^{1 / 2}\right)^{1-2 \beta} ; 0<x<1 .
$$

Proof. Let us consider the model (1) for the case when $k=2$ and take the parameters as $\alpha_{1}=\alpha+$ $\frac{n}{2}, \alpha_{2}=\alpha_{3}=\beta$ and $\beta_{2}=-\left(\beta+\frac{1}{2}\right)$. Now the density of $\nabla_{2, n}^{2}$ as a marginal density of $x_{1}$, can be obtained as the following:

$$
g\left(x_{1}\right)=\frac{1}{2^{2 \beta} B(2 \alpha+n-1,2 \beta)} x_{1}^{\alpha+\frac{n}{2}-1}\left(1-x_{1}\right)^{2 \beta-1}{ }_{2} F_{1}\left(\beta, \beta+\frac{1}{2} ; 2 \beta ; 1-x_{1}\right) ;
$$


for $0<x_{1}<1$ and zero elsewhere, where $B(\alpha, \beta)$ is the beta function.

Alternatively, we can obtain the density of $\nabla_{2, n}^{2}$ by using Meijer's G-function given in Equation (4). Then the density function obtained has the form:

$$
g\left(x_{1}\right)=\frac{1}{2 B(2 \alpha+n-1,2 \beta)}\left(x_{1}^{1 / 2}\right)^{2 \alpha+n-3}\left(1-x_{1}^{1 / 2}\right)^{2 \beta-1} ; 0<x_{1}<1
$$

and zero elsewhere.

Since the density function is unique, Equations (5) and (6) must be equal. Hence the result follows.

Since Equation (5) is a probability density function we obtain the following relation:

\section{Proposition 3.}

$$
\int_{0}^{1} x^{[(2 \alpha+n-1)-1] / 2}(1-x)^{2 \beta-1}{ }_{2} F_{1}\left(\beta, \beta+\frac{1}{2} ; 2 \beta ; 1-x\right) \mathrm{d} x=2^{2 \beta} B(2 \alpha+n-1,2 \beta) .
$$

Many multivariate procedures based on random samples from multivariate normal populations can be interpreted as the study of the distribution of $\nabla_{k, n}^{2}$. The exact distribution of likelihood ratio criteria for testing hypothesis in MANOVA, MANCOVA, multivariate regression analysis etc can be obtained as a special case of distribution of $\nabla_{k, n}^{2}$. Thomas and Thannippara [5,6] expressed the density of the above mentioned likelihood ratio criteria in terms of the marginal distribution of the generalized type-1 Dirichlet model given in Equation (1) with specific set of parameter values. The density of the likelihood ratio criterion $U_{k}, m, n$ for $k=4$ is obtained to be the following:

$$
g(x)=\frac{\Gamma(n+m-1) \Gamma(n+m-3)}{2 \Gamma(n-1) \Gamma(n-3) \Gamma(2 m)}\left(x^{1 / 2}\right)^{n-3}\left(1-x^{1 / 2}\right)^{2 m-1}{ }_{2} F_{1}\left(m+2 ; m ; 2 m ; 1-x^{1 / 2}\right)
$$

for $0<x<1, n \geq 4$ and zero elsewhere. Since Equation (7) is a probability density function we obtain the following relation:

\section{Proposition 4.}

$$
\int_{0}^{1}\left(x^{1 / 2}\right)^{n-3}\left(1-x^{1 / 2}\right)^{2 m-1}{ }_{2} F_{1}\left(m+2 ; m ; 2 m ; 1-x^{1 / 2}\right) \mathrm{d} x=\frac{2 \Gamma(n-1) \Gamma(n-3) \Gamma(2 m)}{\Gamma(n+m-1) \Gamma(n+m-3)} .
$$

Note that the evaluation of a $G$-function involves evaluation of residues at poles of different orders. Hence in such cases we may end up with psi, gamma or zeta functions. The above results are useful in evaluating the definite integrals involving $G$-functions of the type $G_{k, k}^{k, 0}(\cdot)$.

Conflicts of Interest: The authors declare no conflict of interest.

\section{References}

1. Thomas, S.; George, S. A review of Dirichlet distribution and its generalizations. J. Indian Soc. Probab. Stat. 2004, 8, 72-91.

2. Mathai, A.M.; Haubold, H.J. Special Functions for Applied Scientists; Springer: New York, NY, USA, 2008.

3. Mathai, A.M. An Introduction to Geometrical Probability: Distributional Aspects with Applications; Gordon and Breach Science Publishers: Amsterdam, The Netherlands, 1999.

4. Thomas, S.; Mathai, A.M. p-Content of a p-parallelotope and its connection to likelihood ratio statistic. Sankhya A 2009, 71, 49-63.

5. Thomas, S.; Thannippara, A. Distribution of the LR criterion $U_{p, m, n}$ as a marginal distribution of a generalized Dirichlet model. Statistica 2008, 68, 375-390. 
6. Thomas, S.; Thannippara, A. Distribution of $\Lambda$-criterion for sphericity test and its connection to a generalized Dirichlet model. Commun. Stat. Simul. Comput. 2008, 37, 1384-1394.

(C) 2015 by the author; licensee MDPI, Basel, Switzerland. This article is an open access article distributed under the terms and conditions of the Creative Commons by Attribution (CC-BY) license (http:/ / creativecommons.org/licenses/by/4.0/). 